\title{
ON PROJECTIVE DEGENERATIONS OF VERONESE SPACES
}

\author{
EDOARDO BALLICO \\ Department of Mathematics, University of Trento \\ I-38050 Povo (TN), Italy \\ E-mail: (bitnet) ballico@itncisca,ballico@itnvax.science.unitn.it
}

\begin{abstract}
Here we give several examples of projective degenerations of subvarieties of $\mathbb{P}^{t}$. The more important case considered here is the $d$-ple Veronese embedding of $\mathbb{P}^{n}$; we will show how to degenerate it to the union of $d^{n} n$-dimensional linear subspaces of $\mathbb{P}^{t} ; t:=(n+d) /(n ! d !)-1$ and the union of scrolls. Other cases considered in this paper are essentially projective bundles over important varieties. The key tool for the degenerations is a general method due to Moishezon. We will give elsewhere several applications to postulation problems and to embedding problems.
\end{abstract}

We work over the complex number field. The aim of this paper is to give some key examples of projective degenerations of important subvarieties of $\mathbb{P}^{t}$. The more important case considered here is the $d$-ple Veronese embedding of $\mathbb{P}^{n}$; we will show how to degenerate it to the union of $d^{n} n$-dimensional linear subspaces of of $\mathbb{P}^{t} ; t:=(n+d) /(n ! d !)-1$ and to the union of scrolls (see Theorem 0.1 and the discussion in Remark 2.1 for a more precise statement). Other cases considered in this paper either explicitly (see parts (i) and (ii) of Theorem 0.1 and Theorem 3.1) or implicitly (see Remark 3.2) are essentially projective bundles over important varieties. We will give elsewhere several applications will be a partial extension of the results on non complete embedding proved in [BE] (in which it was considered the two-dimensional case). For another application, see Remark 2.2. As in $[\mathrm{BE}]$, section 1 , the key tool for the degeneration is a general method due to Moishezon (see $[\mathrm{M}]$ ).

Here is the main result of this paper.

Theorem 0.1. Let $u: \mathbb{P}^{n} \rightarrow \mathbb{P}^{t}, t+1=(n+d) /(n ! d !)$, be the d-ple Veronese embedding of $\mathbb{P}^{n}$. Then for each of the cases $(i),($ ii $)$, (iii) listed below there is an integral family $\left\{g_{t}\right\}_{t \in T}$ of automorphisms of $\mathbb{P}^{t}$ with as flat limit of $g_{t}\left(u\left(\mathbb{P}^{t}\right)\right)$ in the Hilbert scheme $\operatorname{Hilb}\left(\mathbb{P}^{t}\right)$ of $\mathbb{P}^{t}$ the following connected union $\Pi$ of varieties:

1991 Mathematics Subject Classification: Primary 14D15; Secondary 14M99, 14 N05.

The paper is in final form and no version of it will be published elsewhere. 
(i) $\Pi$ as connected union of $d$ scrolls $Y(n, i), 1 \leq i \leq d$ with the $\operatorname{deg}(Y(n, 1))=1$, $\operatorname{deg}(Y(n, i))=i^{n}-(i-1)^{n}$, for $i>1, Y(n, i)$ isomorphic as abstract variety to $\mathbb{P}^{n}$ if $i=1$ and to the pojective bundle $\mathbb{P}(\mathbb{O} \oplus \mathbb{O}(-1))$ over $\mathbb{P}^{n-1}$ if $i>1$. Furthermore, for every $i>$ $1, Y(n, i) \cap Y(n, i+1)$ is the $i$-ple Veronese embedding of $\mathbb{P}^{n-1}$ and $Y(n, i) \cap Y(n, i+2)=\infty$.

(ii) $\Pi$ is the connected union of $d ! /(i ! /(d-i) !)$ copies of subvarieties $X(n, d, i), 1 \leq i \leq$ $\min (n, d)$ such that $\operatorname{deg}(X(n, d, i))=n ! /(n-i+1)$ !, and as abstract varieties $X(n, d, i)$ is isomorphic to $\mathbb{P}^{n-i+1} \times \mathbb{P}^{1} \times \ldots \times \mathbb{P}^{1}\left(i-1 \mathbb{P}^{1}\right.$ 's as factor $)$. Furthermore, the union of all the varieties $X(n, j, i), 1 \leq i \leq \min (j, n)$, is a projective degeneration of $Y(n, i)$.

(iii) $\Pi$ is a connected union of $d^{n} n$-dimensional linear subspaces of $\mathbb{P}^{t}$. Furthermore, for all $n, d, i$, the union of $\operatorname{deg}(X(n, d, i))$ of these linear spaces is a degeneration of one of the varieties $X(n, d, i)$ (and every linear space arises in this way as a degeneration of exactly one of the varieties considered in $(i i))$.

Theorem 0.1 will be proved in section 2 . In section 1 we will describe a general method of Moishezon ([M]) to obtain projective degeneration. In section 3 we will consider more general cases, but being able to give much less informations on the reduced schemes arising after the degeneration. Except very easy cases, we can say only that this reduced scheme is equidimensional and its irreducible components are linear subspaces of the ambient projective space.

The author was partially supported by MURST and GNSAGA of CNR (Italy). This paper is in final form and no part of it will be published elsewhere.

1. In this section we will describe a method due to Moishezon (see [M], section 2) to obtain projective degenerations.

The following definition was given in [M], Def.1 of section 2.

Definition 1.1. Let $X, Y$ be two complex projective reduced algebraic varieties and $g: X \rightarrow \mathbb{P}^{L}, h: Y \rightarrow \mathbb{P}^{M}$ be two embeddings. We say that $(X, g)$ is a projective degeneration of $(Y, h)$ if there is a family $\left\{Y(t), t \in \mathbb{C}^{1}\right\}$ with embeddings $h_{t}: Y(t) \rightarrow \mathbb{P}^{N}$, $N \geq \max (M . L)$ (depending algebraically on $\mathrm{t}$ ), an integral family of automorphisms $\left\{g_{t}\right\}_{t \neq 0}$, projections $\pi_{L}: \mathbb{P}^{N} \rightarrow \mathbb{P}^{L}, \pi_{M}^{\prime}: \mathbb{P}^{N} \rightarrow \mathbb{P}^{M}$, such that $Y(0) \cong X, \pi_{L}$ is defined on $h_{0}(Y(0)), \pi_{L \mid} h_{0}(Y(0))$ inducing an isomorphism onto $g(X)$, and for every $t \neq 0 \pi_{M}^{\prime}$ defined on $Y(t), Y(t) \cong Y$ and $\pi^{\prime}{ }_{M} h_{t}=g_{t} h$.

Of course, changing the embedding $h_{t}$ we may assume in Definition 1.1 that $g_{t}$ is the Identity for every $t \neq 0$. However, the form of 1.1 just written make clear why such projective degenerations imply statements like Theorem 0.1.

Although we will not need it, we would like to point out the following variation of definition 1.1 in which, instead of $\mathbb{C}^{1}$ we take an integral smooth affine curve, $\Delta$, and $0 \in \Delta$ and in general make the same change in all the definitions and discussions considered in this section. Everything works in the setting, too.

We will make the following assumption and fix the corresponding notations.

Assumption $\left(A_{1}\right)$ : Let $Z$ be a reduced variety, $Z=A \cup B, A, B$ subvarieties of $Z$ (with $Z=A$ allowable even if $B \neq \infty$ ) and let $g: Z \rightarrow \mathbb{P}^{N-1}$ be a projective embedding. 
Following $[\mathrm{M}]$, section 2, we say that $(Z, A, B, g)$ satisfies Assumption $\left(A_{1}\right)$ if the following is true:

(i) $B$ is defined by a sheaf of principal ideals;

(ii) the ideal of $C=A \cap B$ on $A$ is equal to the restriction to $A$ of the ideal of $B$ on $Z$;

(iii) $C$ does not have common irreducible components with $A$. Clearly $C$ is a Cartier divisor on $A$. Set $W:=Z \times \mathbb{C}^{1}, Z_{0}:=Z \times\{0\}, A_{0}:=A \times\{0\}, C_{0}:=C \times\{0\}$. Let $p: W \rightarrow$ $\mathbb{C}^{1}, q: W \rightarrow Z$ be the projections , $B^{\prime}:=q^{-1}(B)$. Let $\phi: W " \rightarrow W$ be the monoidal transformation of $W$ with the center $B_{0}, B^{\prime \prime}$ and $Z$ " the strict transform of $B^{\prime}$ and $q^{-1}(Z)$ in $W^{\prime \prime}$. Let $G \in \operatorname{Pic}(Z)$ be very ample. Set $G^{\prime}:=\left(\phi^{*} q^{*}(G)-\left[\phi^{-1}\left(B_{0}\right)\right]\right) \in \operatorname{Pic}\left(W^{\prime \prime}\right)$. Let $\alpha_{i}, 1 \leq j \leq M$, be a basis for $H^{0}\left(A, \mathbb{O}_{A}((G \mid A)-C)\right)$. Let $\alpha{ }_{i} \in H^{0}(Z ", G \mid Z$ ") be induced by $\alpha_{i}$. Fix $\phi \in H^{0}\left(Z, G^{\prime}\right)$. Let $\phi_{j}, 1 \leq j \leq N$, be a basis for $H^{0}(Z, G)$ and let $\phi^{\prime \prime}{ }_{j}, 1 \leq j \leq N$, be the corresponding elements of $H^{0}\left(Z^{\prime \prime}, G^{\prime} \mid Z^{\prime \prime}\right)$. We make the following assumption $\left(A_{2}\right)$ :

Assumption $\left(A_{2}\right) \cdot \mathbb{O}_{A}((G \mid A)-C)$ is spanned by its global sections. Let $f: Z$ " $\rightarrow$ $\mathbb{P}^{N+M-1}$ be the map sending $P$ to $\left(\Phi{ }_{1}(P): \ldots: \Phi{ }_{N}(P): \alpha{ }_{1}(P): \ldots: \alpha_{M}(P)\right)$. Let $\pi_{N}: \mathbb{P}^{N+M-1} \rightarrow \mathbb{P}^{N-1}$ (resp. $\pi_{M}^{\prime}: \mathbb{P}^{N+M-1} \rightarrow \mathbb{P}^{M-1}$ ) be the rational projection on the first $N$ (resp. last M) homogeneous ccordinates. Using assumption $\left(A_{2}\right)$ it is easy to check that $f$ is a regular morphism with the following properties:

(i) for every $t \in \mathbb{C}^{1} \backslash\{0\} \pi_{N} f \mid \phi^{-1} p^{-1}(t)$ is induced by the given embedding of $Z \times\{t\}$ into $\mathbb{P}^{N-1}$ corresponding to $G$;

(ii) $\pi_{N} f \mid B_{0}^{\prime}$ is induced by the given embedding of $B_{0}$ into $\mathbb{P}^{N-1}$ induced by $G \mid B$;

(iii) $f \mid\left(B{ }_{0} \backslash\left(B{ }_{0} \cap A_{0}\right)\right)$ is an embedding;

(iv) $\pi_{M}^{\prime} f \mid A_{0}$ is the restriction of the morphism $A \rightarrow \mathbb{P}^{M-1}$ which is induced by $H^{0}(A,(G \mid A) \backslash C)$. Set $Z^{\prime}:=f \phi^{-1}\left(Z_{0}\right), A^{\prime}:=f\left(A_{0}^{\prime}\right), B^{\prime}:=f\left(B^{\prime \prime}{ }_{0}\right)$, and $g^{\prime}: Z^{\prime} \rightarrow \mathbb{P}^{N+M-1}$ the corresponding embedding. As in $[\mathrm{M}]$ we will say that $\left(Z^{\prime}, A^{\prime}, B^{\prime}, g^{\prime}\right)$ is obtained from $(Z, A, B, g)$ by construction $(D)$. It is clear that the pair $\left(Z^{\prime}, g^{\prime}\right)$ obtained from $(Z, g)$ with construction $(D)$ is a projective degeneration of $(Z, g)$.

2. In this section we will prove Theorem 0.1. In Remark 2.1 we will discuss briefly the structure of the intersections of the irreducible component of $\Pi$ for each of the cases (i), (ii) and (iii) arising in the statement of 0.1. In Remark 2.2 we will show how to obtain from Theorem 0.1 projective degenerations of other varieties and how to use the degeneration obtained to study the original varieties; in the last few years these varieties were the subject of several papers from several mathematicians.

Pro of (of Theorem 0.1) We will apply several times the construction (D) described in section 1. At each step of its application we will need to check (or leave the easy check to the reader) that we may do the construction even if in previous steps we applied it or equivalently that we may do do simultaneously all the degenerations described in the uses of construction (D). First, we will make the degenerations needed to prove part (i) of 0.1. Then we will show how to degenerate (again using the construction (D)) each of the irreducible components of the reduced scheme $\Pi$ arising in part (i) of 0.1 to the union of irreducible components of the reduced scheme (called again $\Pi$ ) described in part (ii). 
Then we will show how to degenerate each of the components of the scheme $\Pi$ of part (ii) to union of linear spaces to obtain part (iii). To check at each step what are the varieties arising in the degeneration by construction (D) we will use double induction on $n$ and $d$. Hence we will use an essential way the statements for the case $n=2$ proved briefly in [M], section 3. These statements and proofs are explained in detail for $n=2, d=3$ in [MT]. However for $n=2$ the case $d>3$ are very similar to the case $\mathrm{d}=3$ and even it would be possible to prove the case $n=2, d>3$ just formally from the case $n=2, d=3$. Hence the results in $[\mathrm{M}]$, section 3., have a full proof in print. In particular, unless otherwise stated we assume $n \geq 3$. We divide the proof into 3 main parts (i), (ii), (iii) corresponding respectively to cases (i), (ii) and (iii) of the statement of 0.1. Part (i) (resp. (ii)) will be divided into 3 steps (resp. 2 steps).

Proof (of part (i)):

Step $(i 1)$. As in $[\mathrm{M}]$ we start with $V_{n, d}:=u\left(\mathbb{P}^{n}\right) \subset \mathbb{P}^{t}$ and apply the construction (D) taking $Z=A=V_{n, d}, B=u(H), H$ hyperplane of $\mathbb{P}^{n}$. We find a projective degeneration of $V_{n, d}$ to $V_{n, d-1} \cup T_{d}$ for some $T_{d}$. Then we repeat $d-1$ times the construction, each time for a lower integer $d$, and find a projective degeneration of $V_{n, d}$ to a union $T_{1} \cup T_{2} \cup \ldots \cup T_{d}$ with $T_{1}$ n- dimensional linear space.

Step (i2). Here we will check that for every $k \leq d$ we have $T_{k}=Y(n, k)$. First, by the case $n=2$ proved in $[\mathrm{M}]$ we know that each $T_{k}$ is a fiber space with fiber $\mathbb{P}^{1}$ over some variety. We identify this variety as $\mathbb{P}^{n-1}$ and its embedding in $\mathbb{P}^{t}$ using the case $n-1$ assumed by induction. Since the base variety $\mathbb{P}^{n-1}$ has trivial Brauer group, this fibration is the projectivization $\mathbb{P}(E)$ of a rank 2 vector bundle $E$ on $\mathbb{P}^{n-1}$. By the case $n=2$ considered in $[\mathrm{M}]$, the splitting tipe of the restriction of $E$ to every line is the same: up to a twist it is $0,-1$. By a theorem on uniform vector bundles $([\mathrm{VdV}])$ if $n-1>2$ $E$ is the direct sum of two line bundles (which are, up to the same twist, of degree 0 and -1 ), while if $n-1=2$ either $E$ splits in the same way or $E \cong T \mathbb{P}^{2}(-2)$ (up to the same twist). But we may assume to have proven inductively for every $\mathrm{n}$ the existence and uniformity of $E$. Since the bundle $E$ arising for $V_{n-1, a}$ is the restriction to a hyperplane of the bundle arising for $V_{n, a+1}$, by the case $n=4$ we see that even if $n=3$ the bundle $E$ splits.

Step (i3). By construction $Y(n, i) \cap Y(n, i+1)$ is what claimed in 0.1. Since the two sections of $Y(n, i+1) \rightarrow \mathbb{P}^{n-1}$ at which $Y(n, i)$ and $Y(n, i+2)$ are glued (i.e. the section with normal bundle of degree -1 and the section with normal bundle of degree 1) are disjoint, we have $Y(n, i) \cap Y(n+2)=\vee$ for every i.

Proof (of part (ii)):

Step (ii1). We will apply a few degeneration to each $Y(n, i), i>1$, leaving to the reader easy checking that we may apply the degenerations of $Y(n, i)$ for all i simultaneously. Hence we fix $Y(n, i)$ with $i>1$. First by a blowing up of a fiber $\mathbb{P}^{1}$, of the projection $Y(n, i) \rightarrow \mathbb{P}^{n-1}$ we reduce to the following situation. We have a reduced scheme $T$ and projection $m: T \rightarrow \mathbb{P}^{n-1}$ (with $\mathbb{P}^{n-1}$ embedded by the i-ple Veronese embedding). $T$ has two irreducible components, $W$ and $W^{\prime}$ with $m\left(W^{\prime}\right)$ a point, $W^{\prime} \cong \mathbb{P}^{n}, W^{\prime}$ embedded as a linear space. $W \cong \mathbb{P}^{n-1} \times \mathbb{P}^{1}, m \mid W$ is the first projection and the fibres of $m \mid W$ are embedded as lines. 
Step (ii2). By step (ii1) it is sufficient to analyze the easy case of the degeneration of $\mathbb{P}^{n-1} \times \mathbb{P}^{1}$ with the first factor embedded by the i-ple Veronese embedding and the second factor embedded as a line. The degenerations of $Y(n, i)$ to the union of all $X(n, d, i)$ is just obtained applying induction on $\mathrm{n}$ to the factor $\mathbb{P}^{n-1}$.

Pro of (of part (iii)). By part (ii) and induction, it is sufficient to degenerate $Q:=$ $\mathbb{P}^{k} \times \mathbb{P}^{1}$ embedded by the complete linear system $H^{0}\left(Q, \mathbb{O}_{Q}(1,1)\right)$ (i.e. each $\mathbb{P}^{k}$ and each $\mathbb{P}^{1}$ is embedded as a linear space) into $\mathbb{P}^{2 k+1}$. With this embeddeng $Q$ is a minimal degree scrool over $\mathbb{P}^{1}$. The case $k=1$ was proved in $[\mathrm{M}]$, section 4 , or (but only for the line bundle $\mathbb{O}_{Q}(1, y)$ ) can be proved exactly as in [BE], Lemma 2.2. Hence we obtain that for every dimension the variety $Q$ may be degenerate to a chain of $k+1,(k+1)$-dimensional linear subspaces $Q(1) \cup \ldots \cup Q(k+1)$ with $Q(i) \cap Q(j)$ a linear subspace of dimension $k+1-|i-j|$ in both $Q(i)$ and $Q(j)$.

Remark 2.1. Let $A$ and $B$ be different irreducible components of the reduced schemes $\Pi$ considered in the statement of 0.1. If we are in part (i) of the statement of 0.1 we know $A \cap B$. Assume that we are in part (ii) of 0.1. By induction on $\mathrm{n}$ the proofs of this part gives the various possibility for $A \cap B$ and even more: it gives for each point $P$ of $\Pi$ what are the components (the number, their degree, and so on) containing $P$, i.e. we know the combinatorian structure of $\Pi$. However the situation is messy. Now assume that we are in part (iii) of 0.1. Again, in principle the proof gives inductively the combinatorial structure of $\Pi$. However the situation is very messy even for low $n$ and we never tried compute it in the general case.

The following remark shows an interesting application of therems 0.1 and 3.1 and of any other theorem on projective degenerations.

Remark 2.2. Take a subscheme $G_{0}$ of the reduced scheme $\Pi$ union of linear spaces obtained in part (iii) of Theorem 0.1. Suppose that we may see $G_{0}$ as flat limit of a family $\left\{G_{u}\right\}_{u \in \Delta \backslash\{0\}}$ of subschemes of $\mathbb{P}^{n}$ which goes to $G_{0}$ under the projective degeneration (call it $\left\{V_{u}\right\}_{u \in \Delta}$ ) inside $\mathbb{P}^{t}$ of the Veronese embedding of $\mathbb{P}^{n}$ to $\Pi$. Let $M_{0}$ (resp. $M_{u}$ ) be the linear span of $G_{0}$ (resp. $G_{u}$ ) in $\mathbb{P}^{t}$. Consider the image $T_{0}$ (resp. $T_{u}$ ) of the projection of $\Pi$ (resp. $\left.V_{u}\right)$ from $M_{0}$ (resp. $M_{u}$ ). We may see $T_{0}$ (resp. $T_{u}$ ) as the image of the blowing up $A_{0}$ of $G_{0}$ in $\Pi$ (resp. $A_{u}$ of $G_{u}$ in $V_{u}$ ) by a suitable linear system. We may see $T_{0}$ as a "limit" of $\left\{T_{u}\right\}_{n \in \Delta \backslash\{0\}}$. For general $u \in \Delta$ many cohomological properties of $A_{u}$ and $T_{u}$ can be controlled by the corresponding cohomological properties of $A_{0}$ and $T_{0}$ using a semicontinuity theorem.

3. In this section we consider other projective degenerations (see Theorem 3.1). These varieties are more general than just Veronese embeddings of projective spaces as in Theorem 0.1 , but we will have very few informations about the limit reduced scheme obtained (essentially only that it is equidimensional, connected and with linear subspaces of the ambient projective space as irreducible components).

The case $a=2, m(1)=m(2)=1$ of the following theorem was proved in $[\mathrm{M}]$, section 4 . 
THEOREM 3.1. Fix integers $a \geq 2,\{m(i)\}_{1 \leq i \leq a},\{r(j)\}_{1 \leq j \leq a}$ with $m(i)>0$ and $r(j)>$ 0 for all $i, j$. Set $T(\boldsymbol{r}):=\mathbb{P}^{m(1)} \times \mathbb{P}^{m(2)} \times \ldots \times \mathbb{P}^{m(a)} ;$ let $\mathbb{O}_{T(\boldsymbol{r})}(\boldsymbol{s})$ be the line bundle on $T(\boldsymbol{r})$ which have degree $r(i)$ on the $i^{\text {th }}$-factor $\mathbb{P}^{m(i)}$. Let $T(\boldsymbol{r}, \boldsymbol{s})$ be the image of $T(\boldsymbol{r})$ by the complete linear system $H^{0}\left(T(\boldsymbol{r}), \mathbb{O}_{T(\boldsymbol{r})}(\boldsymbol{s})\right)$ into $\mathbb{P}^{t}, t+1:=\Pi_{1 \leq i \leq a}((m(i)+r(i)) ! / m(i) ! r(i) !)$. Then $T(\boldsymbol{r}, \boldsymbol{s})$ may be degenerated inside $\mathbb{P}^{t}$ to a configuration of $\operatorname{deg}(T(\boldsymbol{r}, \boldsymbol{s}))(m(1)+\ldots$ $\ldots+m(a))$-dimensional linear spaces.

Proof. (a) By induction on $a$ we reduce to the case $a=2$, say $\mathbb{P}^{u} \times \mathbb{P}^{v}$, in which the first factor is embedded linearly, i.e. to the case $Z:=\mathbb{P}^{u} \times \mathbb{P}^{v}$ embedded by $\mathbb{O}(1, r)$. We apply part (iii) of Theorem 0.1 to the r-ple Veronese embedding of $\mathbb{P}^{v}$. In this way we reduce easily to the case $r=1$. We have to degenerate $\mathbb{P}^{u} \times \mathbb{P}^{v}$ embedded by $\mathbb{O}(1,1)$ to the union of $(u+v) /(u ! v !)$ linear spaces.

(b) (Sketch) We will make a general construction of projective degenerations and apply it to the particular case of $Z$ we need for Theorem 3.1. We see $Z$ as $\mathbb{P}(E)$ with $E$ (trivial) rank $u+1$ vector bundle on $Y:=\mathbb{P}^{v}$. Let $\pi: \mathbb{P}(E) \rightarrow Y$ be the projection. We take a hypersurface $H$ of $Y$ and a vector bundle $Q$ on $E$ which is a quotient of $E \mid H$; call $q: E \rightarrow E \mid H \rightarrow Q$ the associated surjection. For Theorem 3.1 we take as $H$ a hyperplane $\mathbb{P}^{v-1}$ of $Y$ and $Q \cong \mathbb{O}_{H}(1)^{\oplus u}$. Set $F:=\operatorname{Ker}(q)$. By [Ma] F is a rank $u+1$ vector bundle on $Y$; it is even called "the elementary transformation of $F$ along $q$ ". Then we may repeat the construction, even changing the hypersurface. For instance if we take again $H$ as hypersurface and $\operatorname{Ker}((E \mid H) \rightarrow Q)$ as new bundle, there is a surjections $F \rightarrow K$ with $E(-H)$ as kernel $([M])$. Assume $\mathbb{P}(E)$ embedded in a big projective space, $\mathbb{P}$ with $\mathbb{O}_{\mathbb{P}}(1)$ or degree one on the fibres of $\pi$. By Grothendieck's definition of projective space, $\mathbb{P}(Q)$ is a subvariety of $\mathbb{P}(E)$. Let $M$ be the linear space spanned by $\mathbb{P}(Q)$. Geometrically, the composition of the elementary transformation along $q$, taking the projectivization $\mathbb{P}(F)$ and the induced embedding in a projective space corresponds to the linear projection of $\mathbb{P}(E)$ from $M$ into a lower dimensional projective space, J. Call $Z^{\prime}$ the image of $\mathbb{P}(F)$ in $J$ and see $J$ as a linear subspace of $\mathbb{P}$. Inside $\mathbb{P}$ as in the proof of [BE], Lemma 2.2, we obtain a flat family of embeddings $\left\{j_{u}\right\}_{u \in \Delta \backslash\{0\}}$ of $\mathbb{P}(E)$ and a family $\left\{g_{u}\right\}_{u \in \Delta \backslash\{0\}}$, $g_{u} \in \operatorname{Aut}(\mathbb{P})$ with $j_{u}(\mathbb{P}(E))=g_{u}(\mathbb{P}(E))$ and with as flat limit, $Z_{0}$, inside $\mathbb{P}$ at $o \in$ $\Delta$ the union of $Z^{\prime}$ and of the exceptional divisor, $\Gamma$, obtained blowing up $\mathbb{P}(E)$ along $\mathbb{P}(Q)$ and then mapping it to $\mathbb{P}$ with the corresponding linear system, exactly as in construction $(D)$. In particular the fibres of $\Gamma \rightarrow \mathbb{P}(Q)$ are mapped linearly into $\mathbb{P}$. In our situation, this gives a true embedding of $\Gamma$ with fibres embedded linearly and we may apply induction on $v$ since $H=\mathbb{P}^{v-1}$; the starting point $v=1$ of the induction was proved in the proof of part (iii) of Theorem 0.1 (essentially in the same way by induction on $u$ seeing $\mathbb{P}^{u} \times \mathbb{P}^{1}$ as a trivial $\mathbb{P}^{1}$-bundle over $\left.\mathbb{P}^{u}\right)$. Then we may iterate the elementary transformation and the construction with $Z^{\prime}$ inside $J$ (even using a different $H$ ). In our situation we use the same $H$ and apply the elemntary transformation which has $E(-H)$ as kernel. Since in our situation $\mathbb{P}(E(-H)) \cong Z$ in not embedded by $\mathbb{O}(0,1)$, in the flat limit we obtain a contraction, too (look at the case $u=v=1$ of the quadric surface). We can check that there will not be any embedded component in the flat limit taking restrictions and induction on $v$; the case $v=1$ was checked in the proof of part (iii) of Theorem 0.1. 
Remark 3.2. Note that part (b) of the proof of Theorem 3.1 is a general method for projective degenerations and that, when it applies, it gives a geometric understanding of what is the limit obtained using construction $(D)$.

\section{References}

[1] E. Ballico and Ph. Ellia, On projections of ruled and Veronese surfaces, J. Algebra 121 (1989), 477-487.

[2] M. Maruyama, Elementary transformations of algebraic vector bundles, in: Algebraic Geometry - Proceedings La Rabida, 241-266, Lect. Notes in Math. 961, Springer-Verlag, 1983.

[3] B. Moishezon, Algebraic surfaces and the arithmetic of braids, II, in: Algebra and geometry, papers in honor of I. Shafarevich, vol. II, 311-344, Contemporary Math. 44, 1985.

[4] B. Moishezon and M. Teicher, Braid group techniques in complex geometry III: projective degeneration of $V_{3}$, in: Classification of Algebraic Varieties, 313-332, Contemporary Math. 162, 1994.

[5] A. Van de Ven, On uniform vector bundles, Math. Ann. 195 (1978), 245-248. 